ARTíCUlo

\title{
Efecto de la sedimentación en la capacidad de adhesión y desprendimiento de la anémona de mar Anemonia alicemartinae (Cnidaria: Anthozoa) en sustratos duros
}

\author{
Effect of sedimentation on the attachment and detachment capacity of the sea anemone \\ Anemonia alicemartinae (Cnidaria: Anthozoa) on hard substrates \\ Cristóbal Villaseñor-Parada ${ }^{1 *}$, Paula E. Neill ${ }^{2}$ Luis Jara-Rojas ${ }^{1}$ \\ ${ }^{1}$ Facultad de Ciencias, Universidad Católica de la Santísima Concepción, Alonso de Ribera 2850, Concepción, Chile \\ ${ }^{2}$ Colegio Almondale Lomas, Lomas de San Sebastián, Sta. Teresa de los Andes 265, Concepción, Chile \\ *Autor corresponsal: cristobal.villasenor@gmail.com
}

\begin{abstract}
Anemonia alicemartinae is a cryptogenic species on the Chilean coast, where it is an invader (i.e., increases its distribution over time). Attaches to hard substrates in intertidal and subtidal systems from the northern Chilean coast to the Concepción Bay. Apparently, their reproduction is mainly asexual, without a larval phase, so it has been proposed that their dispersion occurs through a mechanism of detachment and re-attachment to the substrate, which can be stimulated by biotic and abiotic conditions. In this work, was evaluated the effect of sand on said dispersion mechanism, for which sediment collectors were installed at 14 sampling points, in the coast of Lirquén, in the southeastern of Concepción Bay (the southern limit of distribution of this species in the SE Pacific). The results indicated that the density of anemones decreases exponentially with the amount of sediment, which corresponded mainly to fine and medium sands (i.e., between 0.125 and $0.5 \mathrm{~mm}$ in diameter). Laboratory experiments indicate that anemones attached to substrates with more sands take less time to detach, while those attached to substrates without sands take three times as long to do so. The results suggest that the anemones could perceive the presence of sand as unfavorable conditions, stimulating the detachment of the individuals, in search of more favorable habitats. The role of sand in long-distance dispersal of the species is also discussed.
\end{abstract}

Key words: Dispersion, invasion, Concepción Bay, Lirquén, Pacific SE

\begin{abstract}
Resumen.- Anemonia alicemartinae es una especie criptogénica en la costa chilena, donde se comporta como invasora (i.e., aumenta su distribución a través del tiempo). Vive adherida a sustratos duros en sistemas intermareales y submareales someros desde el norte de Chile hasta la Bahía de Concepción. Al parecer, su reproducción es principalmente asexual, sin una fase larval, por lo que se ha propuesto que su dispersión ocurre mediante un mecanismo de desprendimiento y re-adhesión al sustrato, el cuál puede ser estimulado por condiciones bióticas y abióticas. En este trabajo se evaluó el efecto de la arena sobre dicho mecanismo de dispersión, para lo cual se instalaron colectores de sedimento en 14 puntos de muestreo, en la localidad de Lirquén, en el extremo sudeste de la Bahía de Concepción (el límite sur de distribución de esta especie en el Pacífico SE). Los resultados indicaron que la densidad de anémonas disminuye exponencialmente con la cantidad de sedimento, el cuál correspondió principalmente a arenas finas y medianas (i.e., entre 0,125 y 0,5 $\mathrm{mm}$ de diámetro). Experimentos realizados en laboratorio indican que anémonas adheridas a sustratos con mayor cantidad de arena tardan menos tiempo en desprenderse, mientras que aquellas adheridas a sustratos sin arena demoran el triple de tiempo en hacerlo. Los resultados sugieren que las anémonas podrían percibir la presencia de arena como condiciones poco favorables, estimulando el desprendimiento de los individuos en busca de hábitats más propicios. También se discute el rol de la arena en la dispersión de la especie a larga distancia.
\end{abstract}

Palabras clave: Dispersión, invasión, Bahía de Concepción, Lirquén, Pacífico SE

\section{INTRODUCCIÓN}

Las especies invasoras son aquellas especies exóticas que, luego de ser introducidas en un nuevo sistema, aumentan significativamente su rango de distribución a través del tiempo (Shigesada \& Kawasaki 1997, Richardson et al. 2000, Falk-Petersen et al. 2006, Blackburn et al. 2011). Uno de los aspectos centrales en el estudio de las especies invasoras consiste en conocer los factores y mecanismos mediante los cuales estas especies pueden colonizar nuevos sitios y formar poblaciones estables (Shigesada \& Kawasaki 1997, Catford et al. 2009, Blackburn et al. 2011). La evaluación de estos factores en los extremos del rango de distribución invadido, ofrece una oportunidad única para determinar las condiciones que restringen y/o favorecen su expansión hacia nuevos sitios, permitiendo identificar los ecosistemas más vulnerables a ser invadidos (Sexton et al. 2009). 
En los ecosistemas marinos del Pacífico SE la anémona de mar Anemonia alicemartinae Häussermann \& Försterra, 2001 (Fig. 1a) es una de las especies que se comporta como invasora (Häussermann \& Försterra 2001, Castilla et al. 2005, Castilla \& Neill 2009). La misma habría expandido su rango de distribución en $c a$. de $1.900 \mathrm{~km}$ de costa durante los últimos 60 años (Häussermann \& Försterra 2001, Castilla $e t$ al. 2005, Castilla \& Neill 2009). Si bien, no existe certeza respecto al origen exótico de $A$. alicemartinae en la costa del Pacífico SE (i.e., especie criptogénica sensu Carlton 2009), es una de las pocas especies marinas en Chile de las que se tienen registros de una expansión reciente en sentido nortesur (Häussermann \& Försterra 2001). Además, análisis moleculares utilizados para analizar el patrón latitudinal de la diversidad genética de la especie apoyan la idea de una expansión reciente (Canales-Aguirre et al. 2015).

En Chile, A. alicemartinae se distribuye desde el extremo norte del país (18 $\left.21^{\prime} \mathrm{S}\right)$ hasta la Bahía de Concepción (36 42 'S) (Häussermann \& Försterra 2001), y observaciones más recientes corroboran su ausencia en localidades más australes (Pinochet et al. 2019). En la Bahía de Concepción es una de las especies más conspicuas, alcanzando densidades de hasta 50 individuos $\mathrm{m}^{-2}$ (Jara 2010) (Fig. 1b). Esta especie vive adherida a sustratos rocosos del intermareal y submareal somero (Häussermann \& Försterra 2001), o bien, sobre macroalgas boyantes (Thiel
\& Gutow 2005, Villaseñor-Parada \& Neill 2011, López et al. 2013). Aunque la especie es dioica, no se han reportado estados larvales, ya que al parecer los machos son infértiles (Häussermann \& Försterra 2001). Esto hace suponer que la reproducción en esta especie sería principalmente asexual (i.e., fisión binaria) (Häussermann \& Försterra 2001), lo que explicaría la baja diversidad y estructuración genética a lo largo de la costa chilena (Canales-Aguirre et al. 2015). Por lo tanto, su exitosa dispersión a lo largo de la costa chilena no ocurriría por medio de fases larvales móviles, sino más bien, mediante el desprendimiento y re adhesión de individuos juveniles y adultos que viven fijos al sustrato (López et al. 2013, Canales-Aguirre et al. 2015).

Considerando tales antecedentes, López et al. (2013) proponen dos mecanismos de dispersión: i) a larga distancia (entre hábitats), que podría facilitar la colonización de nuevos sitios a escalas espaciales más grandes; y ii) a corta distancia (dentro del hábitat), donde el organismo selecciona las condiciones ambientales locales más favorables para su asentamiento. Evidencia experimental sugiere que la dispersión a corta distancia podría estar regulada tanto por factores bióticos (e.g., interacciones agresivas con anémonas nativas; Brante et al. 2019), como abióticos (e.g., desecación en pozas intermareales durante mareas bajas; López et al. 2013).
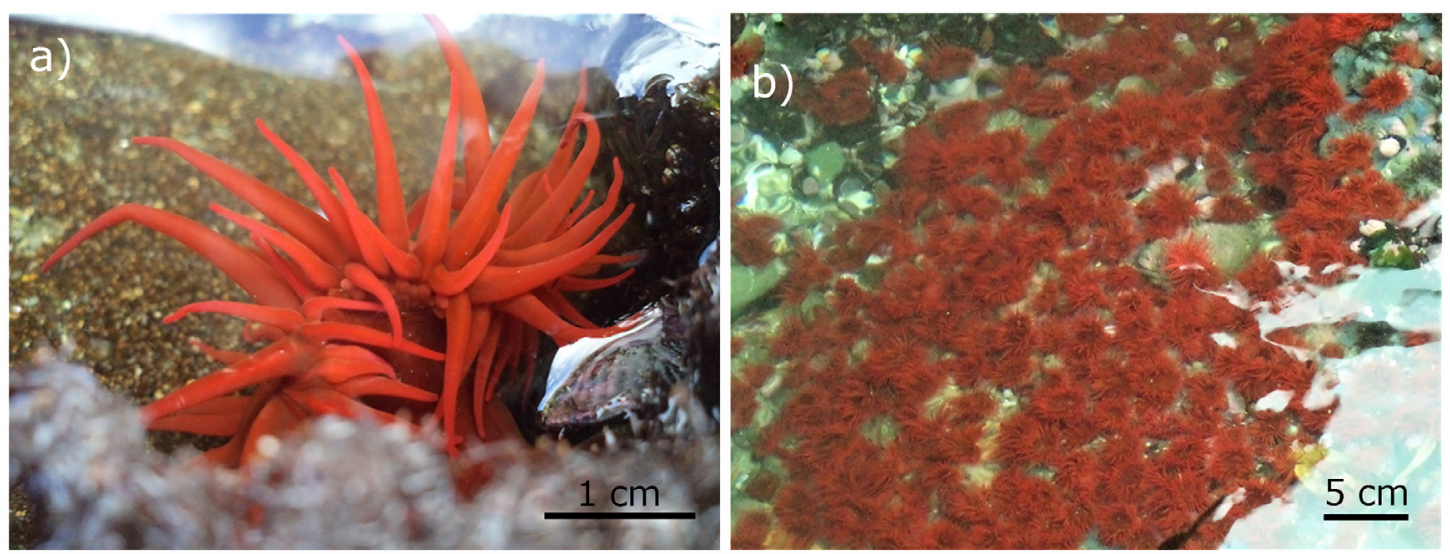

Figura 1. Anemonia alicemartinae en el intermareal rocoso de Lirquén, Concepción, Chile. a) Espécimen vivo, b) Población característica del intermareal. Fotografías de C. Villaseñor / Anemonia alicemartinae in the rocky intertidal area of Lirquén, Concepción, Chile / a) Live specimen, b) Characteristic intertidal population. Photos by C. Villaseñor 
Sin embargo, existen otros importantes factores ambientales cuyo potencial efecto sobre el desprendimiento y re-adhesión al sustrato, aún no han sido abordados. Por ejemplo, a pesar de la alta densidad que alcanzan las poblaciones de $A$. alicemartinae a lo largo de la costa chilena, hay una notable ausencia de individuos en sitios con alta sedimentación (Häussermann \& Försterra 2001). La sedimentación juega un rol clave en la estructuración en comunidades de algas e invertebrados (Littler et al. 1983, D’Antonio 1986, Airoldi \& Virgilio 1998). En cnidarios, algunas especies pueden ser altamente sensibles a la sedimentación (Cairns et al. 2005), mientras que otras pueden generar adaptaciones morfológicas que minimicen su efecto (Littler et al. 1983). En la costa de Lirquén, en el extremo sudeste de la Bahía de Concepción (36 $42^{\circ}$ S, $\left.72^{\circ} 58^{\prime} \mathrm{O}\right)$ (Fig. 2), existen antecedentes de la alta variabilidad espacial y temporal en los patrones de dispersión de sedimentos, asociados principalmente a la acción del oleaje (Ríos et al. 2002). Esta variabilidad espacial, genera un escenario natural en el cuál poder evaluar el efecto de la sedimentación sobre la abundancia de $A$. alicemartinae. Además, la costa de Lirquén corresponde al punto más austral de la distribución de la especie en la costa del Pacífico SE (Pinochet et al. 2019), por lo que experimentos realizados con individuos recolectados en este sector podrían entregar información valiosa relacionada con las condiciones que restringen y/o favorecen su expansión hacia sitios más australes (Sexton et al. 2009). Por lo tanto, el objetivo de este trabajo es evaluar el efecto de la sedimentación sobre la capacidad de desprendimiento y re-adhesión a sustratos duros en individuos de $A$. alicemartinae, discutiendo las potenciales implicancias en el proceso de dispersión de esta especie invasora.

\section{MATERIALES Y MÉTODOS}

\section{SITIO DE ESTUDIO}

Las observaciones fueron realizadas en el intermareal rocoso entre la Punta Lirquén por el norte, y el Puerto de Lirquén por el sur ( $\left.36^{\circ} 42^{\prime} 27^{\prime \prime} \mathrm{S} ; 7^{\circ} 58^{\prime} 36^{\prime \prime} \mathrm{O}\right)$, en el extremo SE de la Bahía de Concepción (Fig. 2). El lugar se caracteriza por la presencia de extensas plataformas y afloramientos rocosos interrumpidos por pequeñas playas de arena (Fig. 3a), y coincide con el límite sur de distribución reportado para Anemonia alicemartinae en la costa del Pacífico SE (Häussermann \& Försterra 2001, Pinochet et al. 2019).

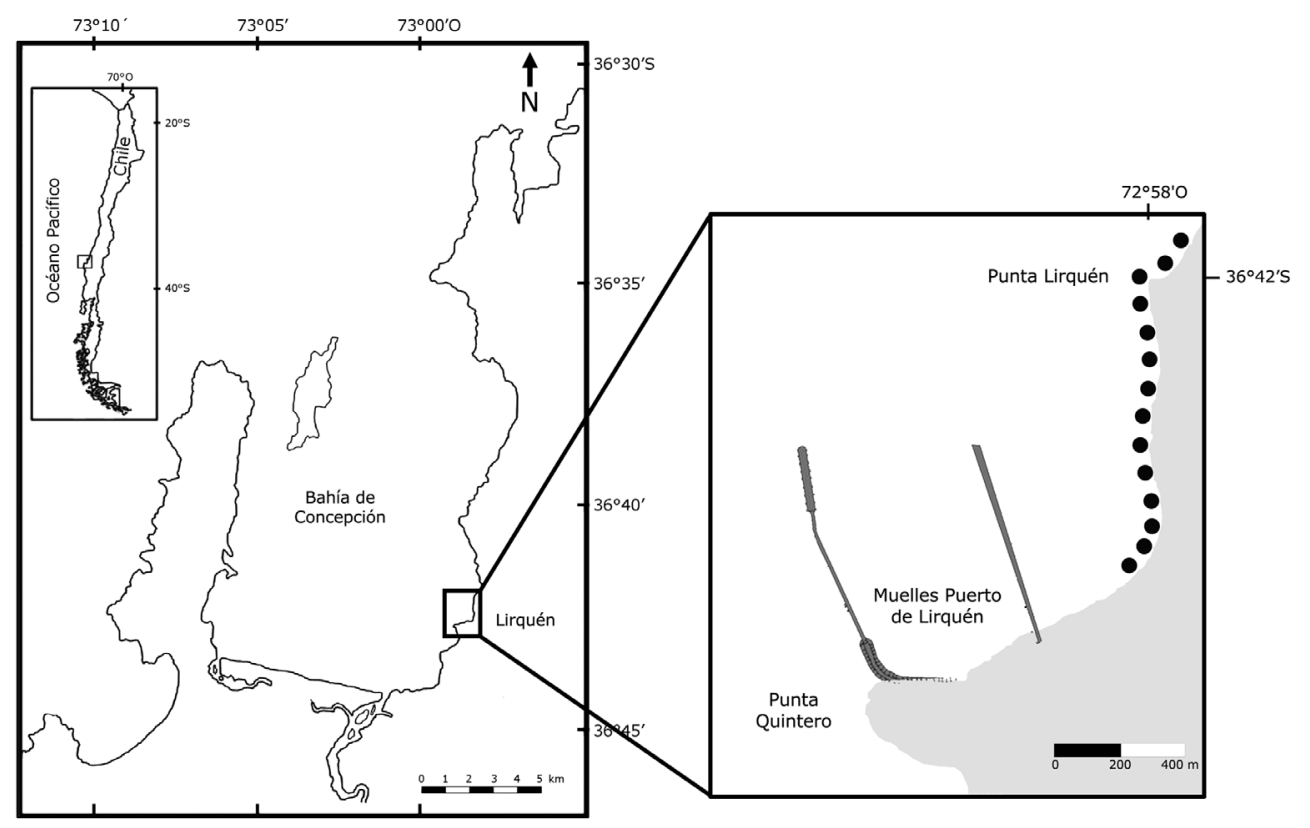

Figura 2. Ubicación de Lirquén en la Bahía de Concepción, y la costa de Chile continental. Círculo negro indica los sitios donde se realizaron las observaciones en terreno y se instalaron los colectores de sedimento / Location of Lirquén in the Concepción Bay and the coast of continental Chile. Black circle indicates the sites where the field observations were made and the sediment collectors were installed 


\section{EFecto de la CANTIDAd de ARENa SObRe la DENSIDAD DE $A$. ALICEMARTINAE EN SISTEMAS INTERMAREALES ROCOSOS}

Durante las bajamares de sicigia de junio 2010, se instalaron 14 colectores de sedimento (Fig. 3b), a lo largo de un transecto paralelo a la línea de costa, de $c a$. de 1,5 $\mathrm{km}$ de longitud (Fig. 2). Estos colectores estaban separados por ca. $100 \mathrm{~m}$ de distancia entre sí, y fueron fuertemente fijados al intermareal rocoso (Fig. 3c), para cuantificar la cantidad de sedimento que llega a la plataforma en un periodo de $24 \mathrm{~h}$. Los colectores fueron construidos con tubos de PVC en forma de "J", con una malla en la abertura delantera para impedir la entrada de algas y otros elementos arrastrados con la marea (Fig. 3b y c). Los colectores fueron construidos siguiendo las proporciones sugeridas en el modelo de Schiel et al. (2006), en donde la longitud del colector es 15 veces el diámetro de la abertura, para así permitir al dispositivo atrapar los sedimentos que caen directamente en los colectores. En nuestro caso, la longitud del colector fue de $112,5 \mathrm{~cm}$ y el diámetro de la abertura fue de $7,5 \mathrm{~cm}$ (Fig. 3b y c).

Al día siguiente, los colectores fueron retirados, no sin antes delimitar un cuadrante de $1 \mathrm{~m}^{2}$ alrededor de cada colector para cuantificar la densidad de A. alicemartinae en cada sitio. Los colectores fueron transportados al laboratorio, donde su contenido de sedimento fue secado en una estufa a temperatura constante $\left(70^{\circ} \mathrm{C}\right)$ por un periodo de $48 \mathrm{~h}$, tras lo cual se obtuvo el peso seco de cada muestra.

Posteriormente, cada muestra de sedimento fue puesta en una tamizadora analítica Restsch As 200, compuesta por 7 tamices de diferente tamaño, por un periodo de $10 \mathrm{~min}$. Esto permitió separar la muestra en diferentes tamaños según la escala granulométrica de Udden-Wentworth (Wentworth 1992). Dicha escala separa el tamaño de grano y/o composición, según el diámetro de la partícula, en las siguientes categorías: limos $(<0,032 \mathrm{~mm})$, arena muy fina (entre 0,032 y $0,125 \mathrm{~mm}$ ), arena fina (entre 0,125 y $0,25 \mathrm{~mm}$ ), arena media (entre 0,25 y $0,5 \mathrm{~mm}$ ), arena gruesa (entre 0,5 y $1 \mathrm{~mm}$ ), arena muy gruesa (entre 1 y $2 \mathrm{~mm}$ ) y gravas $(>2 \mathrm{~mm}$ ). Cada parte del tamizado fue pesado en una balanza electrónica ( $\pm 0,001$ precisión). Los sedimentos fueron guardados en bolsas plásticas para ser utilizados en los experimentos de laboratorio.

La relación entre la densidad de $A$. alicemartinae y la cantidad de sedimento fue evaluada mediante modelos de regresión, utilizando el método de los mínimos cuadrados. Para buscar el modelo con mejor ajuste (e.g., lineal, exponencial) se usó el criterio de selección de Akaike. Los análisis fueron realizados en el programa PAST 4.0 (Hammer et al. 2001).
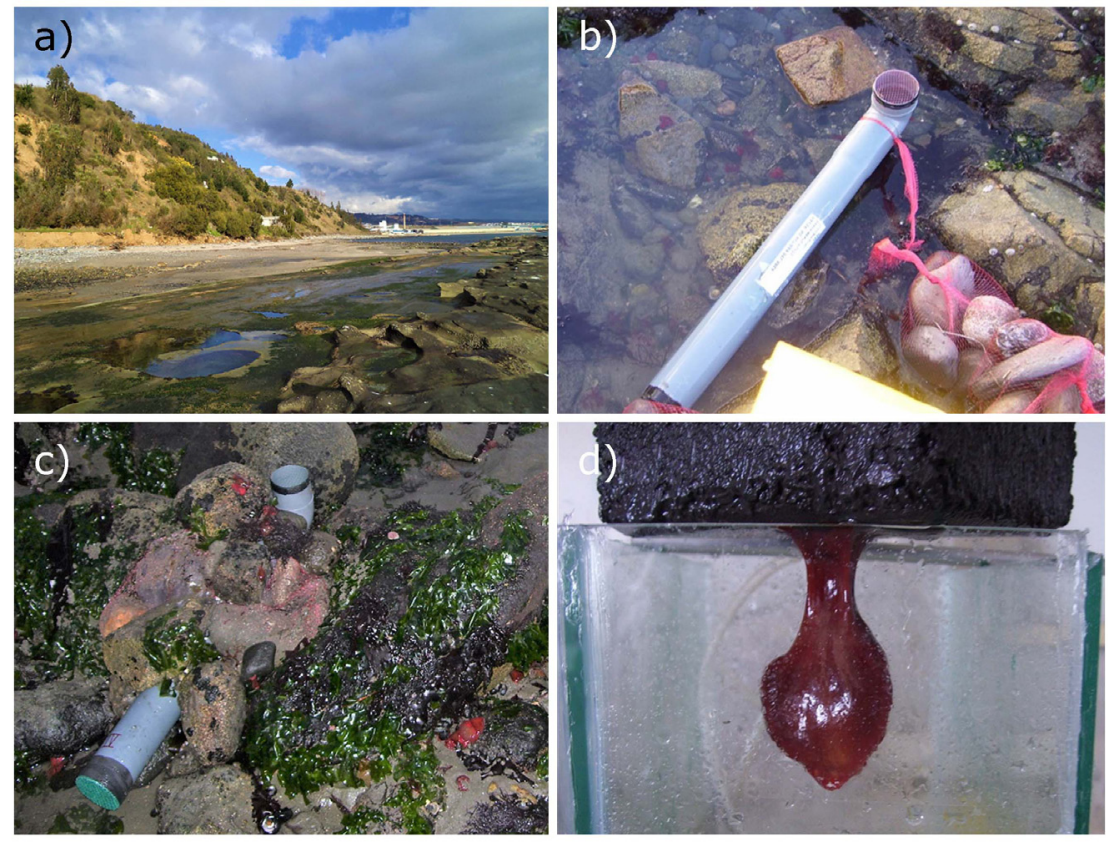

Figura 3. a) Intermareal rocoso de Lirquén donde se realizaron las observaciones en terreno. b) y c) Colector de sedimentos de tipo "J", antes y después de ser instalados en terreno. d) Estimación del tiempo de desprendimiento en los experimentos de laboratorio, volteando individuos de Anemonia alicemartinae adheridos a los sustratos con arenas. Fotografías de C. Villaseñor / a) Rocky intertidal of Lirquén, where observations were made in the field. b) and c) Sediment collectors type " $\mathrm{J}$ " before and after being installed in the intertidal zone. d) Estimation of detachment time in laboratory experiments, turning individuals of Anemonia alicemartinae adhered to substrates with sand. Photos by C. Villaseñor 
EFecto de la CANTIDad de ARena Sobre el DESPRENDIMIENTO DE $\boldsymbol{A}$. ALICEMARTINAE

Para determinar el efecto de la arena sobre el tiempo de desprendimiento de Anemonia alicemartinae, se aplicó un diseño factorial, en el cuál se utilizaron dos tratamientos: i) tamaño de las anémonas (con dos niveles: grandes y pequeñas); y ii) cantidad de sedimento, (con tres niveles: sin arena, con baja cantidad de arena y con alta cantidad de arena). Las combinaciones de los tratamientos fueron dispuestas en 6 acuarios con aireación y temperatura constante $\left(17^{\circ} \mathrm{C}\right)$, cuyo fondo estaba completamente recubierto por ocho adoquines de cemento de $100 \mathrm{~cm}^{2}$.

En terreno, se recolectaron de forma aleatoria alrededor de 200 individuos, los que fueron trasladados al laboratorio. Como la especie se encuentra adherida a rocas fue necesario desprenderlas cuidadosamente para no ocasionar daños en su disco pedal. En el laboratorio, se midió el diámetro del disco basal con un calibrador digital, y su peso húmedo con una pesa analítica ( $\pm 0,01$ precisión). Utilizando estas mediciones, los individuos de $A$. alicemartinae fueron clasificados en dos grupos: pequeños y grandes, mediante el cálculo de los percentiles superiores e inferiores de una distribución de frecuencias. En este trabajo se consideraron como pequeños a los individuos $\leq 1,5 \mathrm{~cm}$ de diámetro basal $\mathrm{y} \leq 2,5 \mathrm{~g}$ de peso, mientras que los grandes correspondían a individuos $\geq 2,5 \mathrm{~cm}$ de diámetro basal $\mathrm{y} \geq 5,3 \mathrm{~g}$ de peso.

Para el tratamiento "cantidad de sedimento", se utilizó arena fina (i.e., entre 0,125 y $0,25 \mathrm{~mm}$ de diámetro), que correspondió al tipo de sedimento más abundante encontrado en las muestras de los colectores (Fig. 4b). La arena utilizada fue la misma que se obtuvo desde los colectores dispuestos en terreno, luego de haber sido secada y tamizada. Sobre cada adoquín que recubría el fondo del acuario, se dispuso de manera uniforme, una cantidad fija de arena, según el nivel del tratamiento: $10 \mathrm{~g}$ para el nivel alta cantidad de arena, y 2,5 g para el nivel baja cantidad de arena.

En cada acuario, se dispusieron de manera uniforme sobre los adoquines, un total de 15 anémonas por un periodo de $24 \mathrm{~h}$ para permitir su adhesión. Luego de transcurrido este tiempo, los adoquines fueron volteados en $180^{\circ}$ dejando la corona de tentáculos en dirección al suelo para estimular el desprendimiento de las anémonas mediante la fuerza de gravedad (Fig. 3d). Se registró el tiempo de desprendimiento para cada individuo.

La evaluación estadística de este experimento se realizó mediante ANDEVA factorial de dos vías (nivel de arena y tamaño de la anémona). Grupos homogéneos fueron identificados mediante la prueba a posteriori de Tukey. Normalidad de los datos fue evaluada mediante la prueba de Shapiro-Wilk, y homogeneidad de varianzas mediante la prueba de Cochran. Para cumplir con los supuestos de normalidad y homogeneidad de varianzas, los datos debieron ser transformados a raíz cuadrada (Zar 1996).

\section{Resultados}

Si bien en todos los sitios muestreados se registró la presencia de Anemonia alicemartinae, su abundancia no fue homogénea a lo largo del transecto, observándose una relación negativa entre la densidad de $A$. alicemartinae y la cantidad de arena (Fig. 4a). El modelo exponencial (Akaike $\mathrm{IC}=522,19$ ) tuvo un mejor ajuste que el modelo lineal (Akaike $\mathrm{IC}=1067,4$ ). Sedimento fue encontrado en once de los catorce colectores. El tamizado analítico identificó las arenas finas y medias como los principales componentes de los sedimentos colectados (Fig. 4b).

Respecto al efecto de la arena fina sobre el desprendimiento de $A$. alicemartinae, al no cumplir los datos con los supuestos de normalidad (Shapiro-Wilk W= $0,96 ; P=0,007)$ y homocedasticidad (Cochran $\mathrm{C}=0,35$; $P=0,008)$, se procedió a aplicar la transformación raíz cuadrada (Normalidad: Shapiro-Wilk W=0,99; $P=0,39$; Homocedasticidad: Cochran $\mathrm{C}=0,38 ; P=0,08)$.

En los experimentos de laboratorio, todas las anémonas lograron fijarse al sustrato (15 individuos por cada combinación de tratamientos) sin importar la cantidad de sedimento en los sustratos. Sin embargo, mientras más arena contenga el sustrato, menor es el tiempo que tardan los individuos en desprenderse. Anémonas adheridas a sustratos con alta cantidad de arena tardaron en promedio media hora en deprenderse, mientras que aquellas adheridas a sustratos con baja cantidad de arena lo hicieron en el doble de tiempo, sin existir diferencias entre individuos grandes y pequeños (Fig. 5). Sin embargo, en sustratos sin arena, individuos pequeños se desprenden más fácilmente que individuos grandes (Fig. 5). 
a)

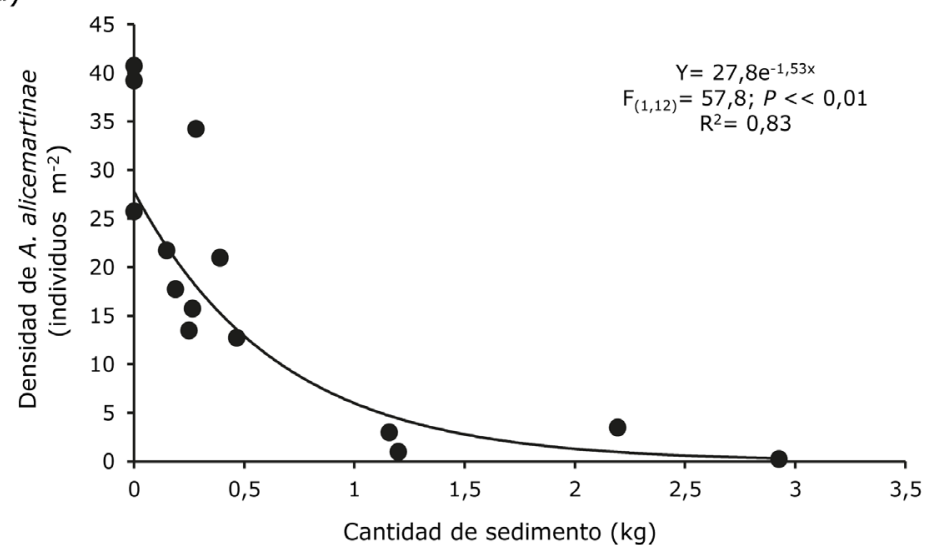

b)

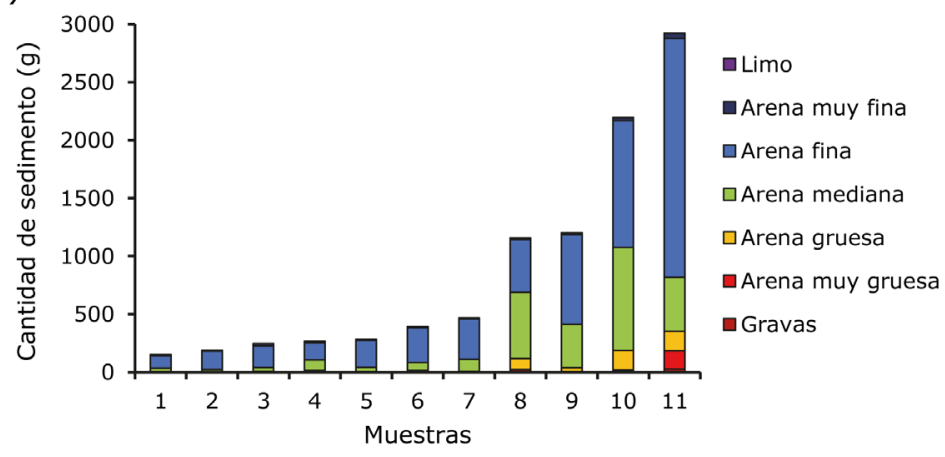

Figura 4. a) Relación entre la densidad de Anemonia alicemartinae (número de anémonas por $\mathrm{m}^{2}$ ) y la cantidad de sedimento encontrada en los colectores instalados en el intermareal rocoso. Resultados del análisis de regresión, se muestran en el extremo superior derecho. b) Cantidad de sedimento encontrados en los colectores instalados en terreno, clasificada por tipo de sedimento, y ordenadas de mayor a menor. Se excluyen los tres colectores en los que no se encontró sedimento / a) Relationship between the density of Anemonia alicemartinae (number of anemones per $\mathrm{m}^{2}$ ) and the amount of sediment found in the collectors installed in the rocky intertidal zone. Results of the regression analysis, shown in the upper right corner. b) Amount of sediment found in the collectors installed on the field, classified by type of sediment, and ordered from highest to lowest. The three collectors in which no sediment was found are excluded

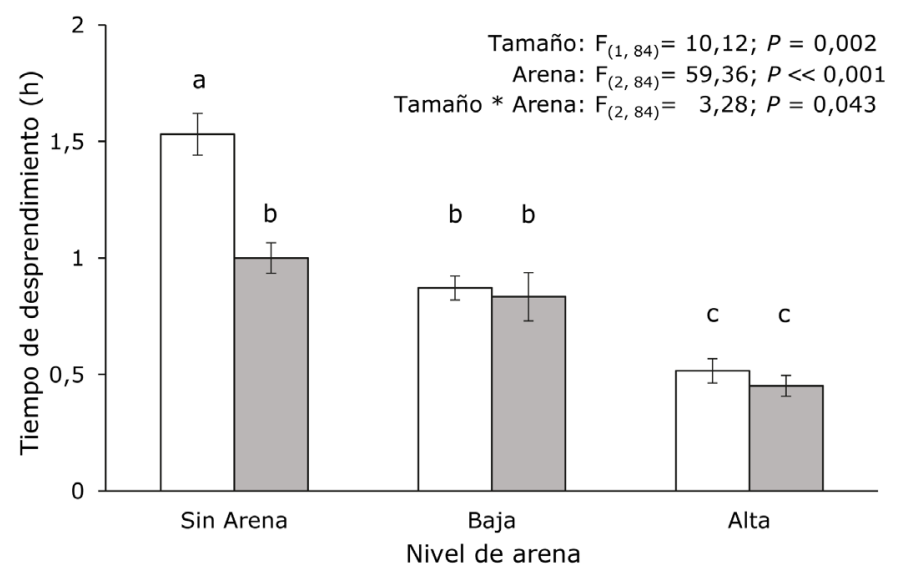

Figura 5. Tiempo de desprendimiento de individuos grandes (columnas en blanco) y pequeños (columnas en gris) de Anemonia alicemartinae (promedio \pm error estándar) adheridos a sustratos con tres niveles de arena. Resultados del ANDEVA factorial se muestran en el extremo superior derecho. Letras sobre las columnas indican grupos homogéneos identificados a partir de la prueba a posteriori de Tukey. Si bien, para cumplir los supuestos de la estadística paramétrica se debieron transformar los datos a raíz cuadrada, la gráfica muestra los datos sin transformar / Detachment time of large individuals (columns in white) and small individuals (columns in gray) of Anemonia alicemartinae (mean \pm standard error) adhered to substrates with three levels of sand. Results of the factorial ANOVA were shown in the upper right corner. Letters above the columns indicate homogeneous groups identified from Tukey's a posteriori test. To fulfill the assumptions of the parametric statistic, the data had to be transformed with the square root, but the graph shows the untransformed data 


\section{Discusión}

A partir de las observaciones realizadas en este trabajo se puede concluir que, aun cuando Anemonia alicemartinae es una de las especies más conspicuas en la costa de Lirquén (Jara 2010), su abundancia no es homogénea en todo el intermareal. Sitios con mayor concentración de arenas en el sedimento presentan una menor densidad de anémonas, coincidiendo con las observaciones realizadas a lo largo de la costa centro norte de Chile (Häussermann \& Försterra 2001). Los mecanismos subyacentes al patrón observado en terreno podrían corresponder a: i) en zonas con mayor sedimentación las anémonas no encuentran sitios libres para su asentamiento; o bien ii) el incremento en la cantidad de arena en los sustratos favorece el desprendimiento de las anémonas ya asentadas. Los resultados de los experimentos de laboratorio apoyan la segunda suposición, ya que todas las anémonas fueron capaces de adherirse en sustratos con diferente cantidad de sedimento, pero mientras más sedimento contenía el sustrato menor tiempo tardaron en desprenderse.

Se ha observado en otros trabajos, que individuos de $A$. alicemartinae sometidos a condiciones adversas aumentan significativamente su frecuencia de desprendimiento como parte de un mecanismo de dispersión a corta distancia (i.e., dentro del hábitat), que les permite escapar a sitios más favorables (López et al. 2013, Brante et al. 2019, Suárez et al. 2020). Tanto en experimentos que simulan las condiciones de desecación en pozas intermareales durante las mareas bajas (López et al. 2013), como en experimentos que simulan un aumento en la temperatura del mar (Suárez et al. 2020), se ha observado un efecto significativo en el aumento de la frecuencia de desprendimiento de las anémonas ante condiciones estresantes. Asimismo, interacciones agresivas con la anémona nativa Phymactis papillosa también estimulan el desprendimiento de $A$. alicemartinae (Brante et al. 2019). Estas interacciones provocan además la aparición de un particular sistema de flotación en su disco pedal (Brante et al. 2019), que también fue observado en los experimentos realizados en este trabajo.

Del mismo modo, la acumulación de arena en los sustratos rocosos puede ser percibida por A. alicemartinae como un potencial riesgo de enterramiento, estimulando el desprendimiento de los individuos en busca de hábitats más propicios para su asentamiento. Este desprendimiento, junto al sistema de flotación generado por la especie ante situaciones adversas (ver Brante et al. 2019) podrían convertir a los individuos desprendidos en entidades dispersantes ("propágulos") a la deriva, capaces de colonizar nuevos ecosistemas mediante reproducción asexual. La aparente ausencia de reproducción sexual en la especie (Häussermann \& Försterra 2001), y por ende, ausencia de una fase larval móvil que garantice la expansión de $A$. alicemartinae hacia nuevos sitios, podría verse sustituida por una exitosa estrategia alternativa de dispersión basada en el desprendimiento y re adhesión al sustrato. Este tipo de estrategias alternativas, han sido observadas en otras especies invasoras en sistemas costeros del Pacífico SE (Villaseñor-Parada et al. 2017, 2018), en donde la liberación de fragmentos reproductivos a la deriva, ha sido identificado como un efectivo mecanismo de expansión de reconocidas macroalgas invasoras como Mastocarpus latissimus (Oróstica et al. 2012) y Codium fragile subp. fragile (Villaseñor-Parada et al. 2013). La baja diversidad genética de $A$. alicemartinae en la costa del Pacífico SE, así como la ausencia de una estructuración en la diversidad de haplotipos a lo largo del gradiente latitudinal, apoyan la idea de una expansión en la costa chilena a partir de reproducción asexual (Canales et al. 2015).

Otro resultado interesante, fue que los individuos grandes adheridos en sustratos sin arena tardaron el triple de tiempo en desprenderse, en comparación con aquellos adheridos a sustratos con sedimento. Esto podría suponer que muelles y estructuras artificiales asociadas a la actividad portuaria podrían constituir hábitats propicios para el establecimiento de $A$. alicemartinae. Sin embargo, trabajos realizados con antelación en la zona no reportan registros de esta anémona en estructuras artificiales del Puerto de Lirquén, a diferencia de otros invertebrados exóticos como Ciona robusta, Bugula neritina y Bugula flabellata, que son importantes componentes de las comunidades incrustantes en los muelles de dicho complejo portuario (Leclerc et al. 2018, 2019). De hecho, en la costa del norte de Chile llama la atención la ausencia de $A$. alicemartinae en estructuras portuarias cercanas a sistemas naturales, en los que ésta anémona es muy abundante (Aguilera 2018). Identificar los factores implicados en la aparente ausencia de A. alicemartinae sobre estructuras portuarias, que son muy utilizadas por otras especies invasoras, resulta una importante interrogante para abordar en futuros trabajos. Estudios que incrementen la complejidad de los experimentos de adhesión/desprendimiento de esta anémona frente a diferentes tipos de sustratos podrían entregar información al respecto.

La ocurrencia de Anemonia alicemartinae en la Bahía de Concepción, es relativamente reciente. Pese a su llamativo color, que le da un aspecto distintivo en sistemas intermareales y submareales (Fig. 1), no se encontraron individuos de $A$. alicemartinae en muestreos realizados en la zona durante 1994 y 1995, siendo reportada por primera vez, de forma esporádica, en muestreos realizados entre 1998 y 1999, y posteriormente con mayores abundancias en el 2001 (Häussermann \& Försterra 2001). Actualmente es una de las especies más conspicuas de los sistemas intermareales y submareales someros en la Bahía de Concepción, la que constituye su actual límite sur de distribución. Evaluar los potenciales factores que favorecen 
o restringen su expansión puede entregar importante información que ayude a comprender la dinámica de expansión de esta especie en Chile continental. En este trabajo, se identifica a la arena como un factor relacionado con el desprendimiento de $A$. alicemartinae de sustratos duros, lo que potencialmente podría favorecer a la dispersión de la especie hacia nuevas regiones.

\section{Agradecimientos}

Este trabajo formó parte de la Tesis de pre grado de L. J-R para optar al título profesional de Biólogo Marino. C. V-P es financiado por Proyecto FONDECYT Postdoctoral $N^{\circ}$ 3210782. Agradecemos a Ecogestión Ambiental Ltda. por facilitar sus instalaciones y equipos de laboratorio para el análisis de sedimentos. Los autores agradecen los comentarios de tres revisores anónimos, cuyas observaciones ayudaron a mejorar este trabajo. Los autores agradecen además la ayuda y comentarios de Lucas Jara Neira, Ilda Riffo Rodríguez (Q.E.P.D), Jaime Villaseñor Fica y Guillermo Villaseñor Bastidas.

\section{LITERATURA CITADA}

Aguilera MA. 2018. Artificial defences in coastal marine ecosystems in Chile: Opportunities for spatial planning to mitigate habitat loss and alteration of the marine community structure. Ecological Engineering 120: 601-610.

Airoldi L \& M Virgilio. 1998. Responses of turf-forming algae to spatial variations in the deposition of sediments. Marine Ecology Progress Series 165: 271-282.

Blackburn TM, P Pyšek, S Bacher, JT Carlton, RP Duncan, V Jarošík, JRU Wilson \& DM Richardson. 2011. A proposed unified framework for biological invasions. Trends in Ecology \& Evolution 26(7): 333-339.

Brante A, R Riera \& P Riquelme. 2019. Aggressive interactions between the invasive anemone Anemonia alicemartinae and the native anemone Phymactis papillosa. Aquatic Biology 28: 127-136.

Cairns SD, V Häussermann \& G Försterra. 2005. A review of the Scleractinia (Cnidaria: Anthozoa) of Chile, with the description of two new species. Zootaxa 1018(1): 15-46.

Canales-Aguirre CB, A Quiñones, CE Hernández, PE Neill \& A Brante. 2015. Population genetics of the invasive cryptogenic anemone, Anemonia alicemartinae, along the southeastern Pacific coast. Journal of Sea Research 102: $1-9$.

Carlton JT. 2009. Deep invasion ecology and the assembly of communities in historical time. In: Rilov G \& JA Crooks (eds). Marine bioinvasions: ecology, conservation, and management perspectives, pp. 439-457. Springer-Verlag, Berlin.

Castilla JC \& PE Neill. 2009. Marine bioinvasions in the Southeastern Pacific: status, ecology, economic impacts, conservation and management. In: Rilov G \& JA Crooks (eds). Marine bioinvasions: ecology, conservation, and management perspectives, pp. 439-457. Springer-Verlag, Berlin.
Castilla JC, M Uribe, N Bahamonde, M Clarke, $\mathbf{R}$ Desqueyroux-Faúndez, H Moyano, N Rozbaczylo, B Santelices, C Valdovinos \& P Zavala. 2005. Down under the southeastern Pacific: marine non-indigenous species in Chile. Biological Invasions 7(2): 213-232.

Catford JA, R Jansson \& C Nilsson. 2009. Reducing redundancy in invasion ecology by integrating hypotheses into a single theoretical framework. Diversity and Distributions 15(1): 22-40.

D'Antonio CM. 1986. Role of sand in the domination of hard substrata by the intertidal alga Rhodomela larix. Marine Ecology Progress Series 27(2): 263-275.

Falk-Petersen J, T Bøhn \& OT Sandlund. 2006. On the numerous concepts in invasion biology. Biological Invasions 8(6): 1409-1424.

Hammer Ø, DA Harper \& PD Ryan. 2001. PAST: Paleontological statistics software package for education and data analysis. Palaeontologia Electronica 4(1): 9. $<$ http://palaeo-electronica.org/2001_1/past/issue1_01.htm>

Häussermann V \& G Försterra. 2001. A new species of sea anemone from Chile, Anemonia alicemartinae n. sp. (Cnidaria: Anthozoa). An invader or an indicator for environmental change in shallow water? Organisms Diversity \& Evolution 1(3): 211-224.

Jara L. 2010. Efecto de la arena sobre la abundancia y readhesión al sustrato de la anémona criptogénica Anemonia alicemartinae en el límite sur de su distribución. Tesis Biología Marina, Facultad de Ciencias, Universidad Católica de la Santísima Concepción, Concepción, 59 pp.

Leclerc JC, F Viard, E González Sepúlveda, C Díaz, J Neira Hinojosa, K Pérez Araneda, F Silva \& A Brante. 2018. Non-indigenous species contribute equally to biofouling communities in international vs local ports in the Biobío region, Chile. Biofouling 34(7): 784-799.

Littler MM, DR Martz \& DS Littler. 1983. Effects of recurrent sand deposition on rocky intertidal organisms: importance of substrate heterogeneity in a fluctuating environment. Marine Ecology Progress Series 11(2): 129-139.

López DN, PAArancibia \& PE Neill. 2013. Potential dispersal mechanisms of the cryptogenic anemone, Anemonia alicemartinae. Revista Chilena de Historia Natural 86(3): 369-372.

Oróstica MH, RD Otaíza \& PE Neill. 2012. Blades and papillae as likely dispersing propagules in Chilean populations of Mastocarpus sp. (Rhodophyta, Gigartinales). Revista de Biología Marina y Oceanografía 47(1): 109-119.

Pinochet J, R Rivera, PE Neill, A Brante \& CE Hernández. 2019. Spread of the non-native anemone Anemonia alicemartinae Häussermann \& Försterra, 2001 along the Humboldt-current large marine ecosystem: an ecological niche model approach. PeerJ 7: e7156. <https://doi. org/10.7717/peerj.7156>

Sexton JP, PJ McIntyre, AL Angert \& KJ Rice. 2009. Evolution and ecology of species range limits. The Annual Review of Ecology, Evolution, and Systematics 40: 415436. 
Schiel DR, SA Wood, RA Dunmore \& DI Taylor. 2006. Sediment on rocky intertidal reefs: Effects on early postsettlement stages of habitat-forming seaweeds. Journal of Experimental Marine Biology and Ecology 331: 158-172.

Shigesada N \& KO Kawasaki. 1997. Biological invasions, 26 pp. Oxford University Press, Tokyo.

Suárez JL, M Hansen, U Urtubia, M Lenz, N Valdivia \& M Thiel. 2020. Season-dependent effects of ocean warming on the physiological performance of a native and a non-native sea anemone. Journal of Experimental Marine Biology and Ecology 522: 151229. < https://doi.org/10.1016/j. jembe.2019.151229>

Thiel M \& L Gutow. 2005. The ecology of rafting in the marine environment. II. The rafting organisms and community. In: Gibson RN, RJA Atkinson \& JDM Gordon (eds). Oceanography and Marine Biology: An Annual Review 43: 279-418.

Villaseñor-Parada C \& PE Neill. 2011. Distribución espacial de epifitos en el talo de la macroalga introducida Codium fragile subsp. tomentosoides en el submareal de Caldera. Revista de Biología Marina y Oceanografía 46(2): 257-262.
Villaseñor-Parada C, EC Macaya, LM Jara \& PE Neill. 2013. Variación temporal y espacial en la producción de gametangios de la macroalga exótica Codium fragile subsp. tomentosoides (Suringar) Hariot (Chlorophyta: Bryopsidales) en el submareal de Caldera. Revista de Biología Marina y Oceanografía 48(1): 213-218.

Villaseñor-Parada C, A Pauchard \& EC Macaya. 2017. Ecología de invasiones marinas en Chile continental: ¿Qué sabemos y que nos falta por saber? Revista de Biología Marina y Oceanografía 52(1): 1-17.

Villaseñor-Parada C, A Pauchard, ME Ramírez \& EC Macaya. 2018. Macroalgas exóticas en la costa de Chile: patrones espaciales y temporales en el proceso de invasión. Latin American Journal of Aquatic Research 46(1): 147165.

Wentworth CK. 1992. A scale of grade and class terms for clastic sediments. Journal of Geology 30: 377-392.

Zar JH. 1996. Biostatistical analysis, 867 pp. Prentice Hall, Englewood Cliffs.

Recibido el 13 de octubre 2020 y aceptado el 23 de mayo 2021 